\title{
Magnetotransport of coupled electron-holes
}

\author{
Y. Naveh ${ }^{1}$ and B. Laikhtman ${ }^{2}$ \\ ${ }^{1}$ Department of Physics and Astronomy, State University of New York, Stony Brook, NY 11794-3800, USA \\ ${ }^{2}$ Racah Institute of Physics, Hebrew University, Jerusalem 91904, Israel
}

\begin{abstract}
The carriers in InAs-GaSb double quantum wells are hybrid "electron-holes". We study the magnetotransport properties of such particles using a two-component Keldysh technique, which results in a semi-analytic expression for the small-field current. We show that zero temperature current can be large even when the Fermi energy lies within the hybridization gap, a result which cannot be understood within a semiclassical (Boltzmann) approach. Magnetic field dependence of the conductance is also affected significantly by the hybridization of electrons and holes.
\end{abstract}

The concept of electrons and holes as the basic quasiparticles in semiconductor physics is usually very useful for the study of transport properties. However, in InAsGaSb heterostructures this concept breaks down. The reason for this is that electrons in the InAs layer can be isoenergetic with holes in the GaSb layer. Then, hybridization occurs between conduction and valence band states [1], and the single-particle excitations in the system are hybrid electron-holes [2]. The effect of hybridization on in-plane transport in the case where the carriers are of the same type was studied in the past [3 [5], and was shown to result in "resistance resonance" if the scattering times of carriers in the two wells are not the same. Hybridization of electrons in two wells also significantly changes their plasmon spectrum [6,7]. However, the case of electron-hole hybridization is very different. For example, in contrast to the electron-electron case, it is not immediately clear what charge is carried by the electronhole excitations. In addition, those excitations follow a strongly non-monotonic dispersion law [2, 8, 9], and a relatively large hybridization gap (later referred to as plain 'gap') is formed. As a result, all transport properties are affected by hybridization even if the scattering rates in the wells are the same.

Much experimental effort has been put into studying these particles. The existence of the gap was confirmed [10,11 and subsequent measurements have revealed more details of the non-monotonic spectrum 12 14. Recent transport measurements 15,16 have shown strong resistance resonance. However, it was shown [15, 16 that the conductance remains finite even when the Fermi level is in the gap. In this regime current carriers are the hybrid electron-holes.

Analyzing theoretically the transport properties of these hybrid particles requires a much more basic approach than in the calculation of the spectrum. This is because natural broadening of levels around the gap plays a crucial role in determining the transport at relevant energies, and, as will be shown below, is the reason for the finite in-gap current. Previous theoretical works studying the transport of particles obeying non-monotonic dispersion laws 17] where based on semiclassical assumptions, and thus could not produce this result. Below we present a full quantum theory for the magnetotransport of hybrid electron-holes. For the sake of definiteness we consider an InAs/GaSb double quantum well system.

Our theory is based on a two component Keldysh technique. In the absence of scattering and at zero electric and magnetic fields, the Hamiltonian for the system takes the form 18

$$
H_{0}=\left(\begin{array}{cc}
E_{e k} & -\left(i k_{x}+k_{y}\right) w \\
\left(i k_{x}-k_{y}\right) w & E_{h k}
\end{array}\right),
$$

where $E_{e k}=\hbar^{2} k^{2} / 2 m_{e}, E_{h k}=E_{g 0}-\hbar^{2} k^{2} / 2 m_{h}, \mathbf{k}$ is the in-plane wavevector, $m_{e, h}$ are the in-plane electron and hole effective masses, and $E_{g 0}>0$ is the energy difference between the first hole and electron levels. Under applied electric and magnetic fields, the Hamiltonian takes the same form as in (1), but with the substitution

$$
E_{\alpha k} \rightarrow E_{\alpha k}-e \mathbf{F} \cdot \mathbf{r}, \quad \mathbf{k} \rightarrow-i \nabla-\frac{e}{\hbar c} \mathbf{A}(\mathbf{r}),
$$

with $\mathbf{F}$ the electric field, $\mathbf{A}$ the vector potential, and $\mathbf{r}$ the in-plane position.

In what follows we calculate the current by first obtaining an explicit expression for the density matrix

$$
\rho\left(\mathbf{r}_{1}, \mathbf{r}_{2}\right)=\int G^{-+}\left(\mathbf{r}_{1}, \mathbf{r}_{2}, E\right) \frac{d E}{2 \pi i},
$$

with $G$ the Keldysh Green's function [19]. Note that $G$ here has 16 components, 4 Keldysh components for each of the 4 terms in the Hamiltonian. It satisfies the Dyson equation 19.

$$
\begin{aligned}
\hat{G}_{01}^{-1} G_{12} & =\sigma_{z}+\sigma_{z} \Sigma_{13} G_{32}, \\
G_{12} \hat{G}_{02}^{-1 *} & =\sigma_{z}+G_{13} \Sigma_{32} \sigma_{z},
\end{aligned}
$$

where $\Sigma$ is the self energy, $\sigma_{z}$ the Pauli matrix with respect to Keldysh indices, and

$$
\hat{G}_{0 j}^{-1}=i \hbar \frac{\partial}{\partial t_{j}}-H_{j j}
$$

in which $\partial / \partial t_{j}$ operates on the $j$ th variable of $G_{12}$.

In the presence of magnetic field $G, \Sigma$, and $\rho$ are not translationally invariant. We therefore work with the translationally invariant functions [20] 


$$
\begin{aligned}
& \tilde{G}\left(\mathbf{r}_{1}-\mathbf{r}_{2}, E\right) \\
& \quad=G\left(\mathbf{r}_{1}, \mathbf{r}_{2}, E-e \mathbf{F} \cdot \mathbf{R}\right) \exp \left[-\frac{i e}{\hbar c} \phi\left(\mathbf{r}_{1}, \mathbf{r}_{2}\right)\right]
\end{aligned}
$$

and similar definitions for $\tilde{\Sigma}$ and $\tilde{\rho}$. Here $\mathbf{R}=\left(\mathbf{r}_{1}+\mathbf{r}_{2}\right) / 2$,

$$
\phi\left(\mathbf{r}_{1}, \mathbf{r}_{2}\right)=-\frac{1}{2} \mathbf{B} \cdot\left(\mathbf{r}_{1} \times \mathbf{r}_{2}\right)+f\left(\mathbf{r}_{1}\right)-f\left(\mathbf{r}_{2}\right),
$$

$\mathbf{B}$ is the magnetic field, and $f(\mathbf{r})$ is the gauge function, defined by $\mathbf{A}(\mathbf{r})=\frac{1}{2} \mathbf{B} \times \mathbf{r}+\nabla f(\mathbf{r})$ [20]. Furthermore, in electric field even $\tilde{G}$ is not translationally invariant. This complication can be neglected if the energy of the carriers and their wavefunctions are not affected appreciably by the field. The restriction on the field is thus

$$
\begin{aligned}
\frac{e F \tau_{e, h}}{\hbar k_{F}}, \frac{e F}{k_{F} \Delta} & \ll 1, \\
I_{\alpha \alpha \mathbf{k}} & =\left(\tilde{\Sigma}_{\alpha \alpha \mathbf{k}}^{r}-\tilde{\Sigma}_{\alpha \alpha \mathbf{k}}^{a}\right) \tilde{G}_{\alpha \alpha \mathbf{k}}^{-+}-\tilde{\Sigma}_{\alpha \alpha \mathbf{k}}^{-+}\left(\begin{array}{l}
\text { bine the various } e-h \text { compograls given by } \\
\text { integrak }
\end{array}\right. \\
I_{\mathbf{k} \alpha \beta} & \left.=\tilde{\Sigma}_{\alpha \alpha \mathbf{k}}^{r} \tilde{G}_{\alpha \beta \mathbf{k}}^{-+}-\tilde{\Sigma}_{\alpha \alpha \mathbf{k}}^{r}\right),
\end{aligned}
$$

with $\tau_{e, h}$ the elastic scattering times of electrons and holes, $k_{F}$ the Fermi wavenumber, and $\Delta=w k_{0}$ the hybridization gap ( $k_{0}$ is defined by $\left.E_{e k_{0}}=E_{h k_{0}}\right)$. When interested in magnetic phenomena this also requires

$$
\frac{e F R_{L} \tau_{e, h}}{\hbar}, \frac{E_{c e, h}}{E_{F}} \ll 1,
$$

with $R_{L}$ the Larmor radius and $E_{c \alpha}=\hbar e B / m_{\alpha} c$. In practice, these limitation are not strong and are applicable to a wide range of experiments.

We now expand Eqs. (4) for all their components, use Eq. (6), and transform into $\mathbf{k}$-space. The resulting equations for the $(-+)$ components of $\tilde{G}$ take forms similar to the evolution equation (4.6) of Ref. [20], but which combine the various $e-h$ components of $G$, and with collision where $\tilde{G}^{r}=\tilde{G}^{--}-\tilde{G}^{-+}, \tilde{G}^{a}=\tilde{G}^{--}-\tilde{G}^{+-}, \tilde{\Sigma}^{r}=$ $\tilde{\Sigma}^{--}+\tilde{\Sigma}^{-+}$, and $\tilde{\Sigma}^{a}=\tilde{\Sigma}^{--}+\tilde{\Sigma}^{+-}$, and where we omitted the explicit dependence on energy for brevity. We also use the convention that if both $\alpha$ and $\beta$ appear in the same equation, they are distinctly different (i.e., if $\alpha=h$ then $\beta=e$ and vice versa).

We now make the physical assumption that scattering in the two wells is not correlated. Formally, this means that $\tilde{\Sigma}$ is diagonal. Also, we follow the usual practice and neglect the small renormalization of the spectrum due to scattering. In this case $\tilde{\Sigma}$ becomes purely imaginary and can be written in the form

$$
\begin{aligned}
& \tilde{\Sigma}_{\mathbf{k}}^{a}(E)=-\tilde{\Sigma}_{\mathbf{k}}^{r}(E)=i\left(\begin{array}{cc}
\Gamma_{e k}(E) & 0 \\
0 & \Gamma_{h k}(E)
\end{array}\right) . \\
& \tilde{G}_{k}^{r}(E)=\tilde{G}_{k}^{a \dagger}(E)=\frac{1}{R(E, k)}\left(\begin{array}{cc}
E-E_{h k}+i \Gamma_{h k} & -w\left(i k_{x}+k_{y}\right) \\
w\left(i k_{x}-k_{y}\right) & E-E_{e k}+i \Gamma_{e k}
\end{array}\right),
\end{aligned}
$$

$\Gamma_{\alpha k}(E)$ are found within the self-consistent Born approximation by writing down the general expression for the self energy,

$$
\tilde{\Sigma}_{\alpha \alpha \mathbf{k}}(E)=\int \tilde{G}_{\alpha \alpha \mathbf{k}-\mathbf{q}}(E) u_{\alpha \mathbf{q}} \frac{d^{2} \mathbf{q}}{(2 \pi)^{2}},
$$

where $u_{\alpha \mathbf{q}}$ are the elastic scattering matrix elements squared. $\tilde{G}_{\alpha \alpha \mathbf{k}}$ is then found by solving the general relation 20]

$$
E \tilde{G}^{r, a}-\frac{1}{2}\left\{H, \tilde{G}^{r, a}\right\}=1+\frac{1}{2}\left\{\tilde{\Sigma}^{r, a}, \tilde{G}^{r, a}\right\},
$$

with $\{A, B\}=A B+B A$. The solution of this equation

with

$$
R(E, k)=\left(E-E_{e k}+i \Gamma_{e k}\right)\left(E-E_{h k}+i \Gamma_{h k}\right)-w^{2} k^{2},
$$

and where we used Eqs. (11) and (11). In the same approximation, substituting Eq. (14) in Eq. (12) gives

$$
\tilde{\Sigma}_{\alpha \alpha \mathbf{k}}^{r}(E)=-i \int \operatorname{Im} K_{11}^{\beta}(E, q) u_{\alpha \mathbf{k}-\mathbf{q}} \frac{d^{2} \mathbf{q}}{(2 \pi)^{2}},
$$

where the family of kernels $K_{p q}^{\alpha}$ are defined by

$$
K_{p q}^{\alpha}(E, k)=\frac{\left[E-E_{\alpha k}-i \Gamma_{\alpha k}(E)\right]^{p}}{\left[R(E, k)^{q}\right]^{*}} .
$$

For short-range scattering $u_{\alpha \mathbf{k}}$, and therefore $\tilde{\Sigma}$ and $\Gamma$, do not depend on $\mathbf{k}$. Its value can be found from Eqs. 16.17) at energies far from the gap, where they are expressed in terms of the relaxation times in non-coupled wells,

$$
\frac{\hbar}{\tau_{\alpha}}=\frac{u_{\alpha}}{2} \int_{0}^{\infty} \delta\left(E-E_{\alpha k}\right) k d k=\frac{m_{\alpha} u_{\alpha}}{2 \hbar^{2}} .
$$

Combining Eqs. (11, 16, 18) results in two self consistent equations for $\Gamma_{e}, \Gamma_{h}$, 


$$
\Gamma_{\alpha}(E)=\frac{\hbar^{3}}{\pi m_{\alpha} \tau_{\alpha}} \int_{0}^{\infty} \operatorname{Im} K_{11}^{\beta}(E, k) k d k .
$$

Next, we substitute Eqs. (11) and (14) into Eq. (10) which results in collision integrals depending only on the $(-+)$ components of $\tilde{G}$ and $\tilde{\Sigma}$. We then linearize the equations for these components with respect to the electric field, using the unperturbed (zero-order) function $\tilde{G}^{-+(0)}=f_{0}(E)\left\langle\tilde{G}^{a}-\tilde{G}^{r}\right\rangle\left[20\right.$, with $f_{0}(E)$ the Fermi function. After the linearization the equations for the Green's functions are integrated with respect to energy.
With the use of Eq. (3) this leads to four transport equations for the components of the density matrix. The solution to these equations is found in a straightforward, though tedious, manner. It is given in terms of the coefficients $\vec{\chi}(k)$, defined by

$$
\begin{aligned}
& \tilde{\rho}_{\alpha \alpha}^{(1)}(\mathbf{k})=\vec{\chi}^{\alpha \alpha}(k) \cdot \mathbf{k}, \\
& \tilde{\rho}_{\alpha \beta}^{(1)}(\mathbf{k})=\vec{\chi}^{\alpha \beta}(k) \cdot \mathbf{k},
\end{aligned}
$$

with $\tilde{\rho}^{(1)}$ the term of $\tilde{\rho}$ linear in $F$. The solution reads

$$
\begin{aligned}
\chi_{x}^{\alpha \alpha}(k) & = \pm 2 e \hbar^{2} F \frac{\omega_{\beta k} \zeta_{\alpha}(k)-\omega_{k} \zeta_{\beta}(k)}{\omega_{e k} \omega_{h k}-\omega_{k}^{2}}, \\
\chi_{y}^{\alpha \alpha}(k) & = \pm \frac{1}{2} \frac{E_{c \beta} \gamma_{k} \chi_{x}^{\beta \beta}(k)-E_{c \alpha} \gamma_{\beta k} \chi_{x}^{\alpha \alpha}(k)}{\gamma_{e k} \gamma_{h k}-\gamma_{k}^{2}}, \\
2 \operatorname{Re} \chi_{x}^{e h} & =\frac{2 m_{r} w^{2} \Gamma}{E_{k}^{2}+\Gamma^{2}}\left\{\xi_{2}^{h}(k)-\xi_{2}^{e}(k)+\frac{1}{\hbar^{2}}\left[\frac{E_{c h} \Gamma_{e}}{2 \bar{\gamma}_{k}^{2}}+\frac{E_{k}}{\Gamma}\right] \chi_{x}^{h h}(k)-\frac{1}{\hbar^{2}}\left[\frac{E_{c e} \Gamma_{h}}{2 \bar{\gamma}_{k}^{2}}+\frac{E_{k}}{\Gamma}\right] \chi_{x}^{e e}(k)\right\}, \\
2 \operatorname{Re} \chi_{y}^{e h} & =0,
\end{aligned}
$$

with the upper sign for $\alpha=e$ and the lower for $\alpha=h$. Here

$$
\begin{gathered}
\zeta_{\alpha}(k)=\xi_{1}^{\alpha}(k)+w^{2} k^{2} \eta(k), \\
\eta(k)=\frac{1}{\pi m_{r}} \int f_{0}(E) \operatorname{Im} K_{02}(E, k) d E+\frac{2 E_{k}}{E_{k}^{2}+\Gamma^{2}} \int f_{0}(E)\left[\frac{1}{\pi m_{e}} \operatorname{Im} K_{12}^{h}(E, k)-\frac{1}{\pi m_{h}} \operatorname{Im} K_{12}^{e}(E, k)\right] d E,
\end{gathered}
$$

$$
\begin{gathered}
\xi_{1}^{\alpha}(k)=\frac{-1}{\pi m_{\alpha}} \int f_{0}^{\prime}(E) \operatorname{Im} K_{11}^{\beta}(E, k) d E, \\
\xi_{2}^{\alpha}(k)=-\frac{e F}{\pi m_{\alpha}} \int f_{0}(E) \operatorname{Im} K_{12}^{\beta}(E, k) d E, \\
\omega_{\alpha k}=4 \gamma_{\alpha k}+\frac{E_{c \alpha}^{2} \gamma_{\beta k}}{\gamma_{e k} \gamma_{h k}-\gamma_{k}^{2}}, \\
\omega_{k}=4 \gamma_{k}+\frac{E_{c e} E_{c h} \gamma_{k}}{\gamma_{e k} \gamma_{h k}-\gamma_{k}^{2}},
\end{gathered}
$$

$\gamma_{k}=w^{2} k^{2} \Gamma /\left(E_{k}^{2}+\Gamma^{2}\right), \gamma_{\alpha k}=\Gamma_{\alpha}+\gamma_{k}, \Gamma=\Gamma_{e}+\Gamma_{h}$, $E_{k}=E_{h k}-E_{e k}, 1 / m_{r}=1 / m_{e}+1 / m_{h}$, and $x$ is in the direction of the electric field.

The current density is given by

$$
\mathbf{j}=\frac{e i}{S \hbar}\langle[H, \mathbf{r}]\rangle
$$

with $S$ the area of the sample. Performing the average over the density matrix $\tilde{\rho}$, and using Eq. (20) results in

$$
\mathbf{j}=\frac{e \hbar k_{F}^{3}}{2 \pi} \int d k\left[\frac{\vec{\chi}^{e e}(k)}{m_{e}}+\frac{\vec{\chi}^{h h}(k)}{m_{h}}+2 \operatorname{Re} \frac{\vec{\chi}^{e h}(k)}{m_{r}}\right] .
$$

The mixing of electrons and holes is affecting both the diagonal contributions to the current [see Eq. 21( $(\mathrm{a}, \mathrm{b})$ ],

and the unusual off-diagonal contribution (the origin of the latter is solely due to this mixing).

Equations (21,29) present a semi-analytic expression for the current at any temperature and for any value of $\Gamma$, provided the conditions (8.9) are met. [The only numerical tasks required in order to calculate the current are the integration over energy in Eqs. (23, 25) and the solution of Eqs. 19]. Results for the sheet conductance at zero magnetic field and zero temperature as a function of Fermi energy are shown in Fig. 1. Magnetoconductance curves for the case where the Fermi level is in midgap are shown in Fig. 2.

Two important conclusions can be drawn from the results shown in Figs. 1,2. First, it is clear that the zerotemperature conductance does not vanish even when the Fermi level is in the gap. This is due to the finite broadening of the levels $\Gamma$. Moreover, as long as the particles are not localized, conductivity is finite even for very small $\Gamma$. This is because the scattering mean free time (proportional to the conductivity) scales as $1 / \Gamma$ while the overlap of levels in the two hybridized bands is linear with $\Gamma$. In the case when $\Gamma \ll \Delta \ll E_{g 0}$, Eqs. (21,29) result in a crude estimate of the conductivity, $g \approx\left(e^{2} / h\right)\left(E_{g 0} / \Delta\right)$. At very small values of $\Gamma / \Delta$ states in the middle of the gap become localized, and the conductivity falls to a 
value close to $e^{2} / h$ (this effect cannot be described by the first Born approximation used in this work). It would be interesting to study the competition between this quantum resistance, usually proportional to $\ln (L / l)$, with $L$ the sample length and $l$ the mean free path, and our hybridization resistance, proportional to $\Delta / E_{g 0}$. An opportunity to study the two terms is unique to our system because $\Gamma$ does not play a role in the conductivity due to states outside the mobility gap. In addition, the logarithmic dependence of the quantum correction on $L / l$ was obtained only for purely metallic systems. In our case the Fermi level is located in the hybridization gap, and this correction may assume a different form. In any case, it is emphasized that our result of finite conductivity in the gap, which is consistent with recent experimental results 15. 16], cannot be understood within a semiclassical Boltzmann theory.

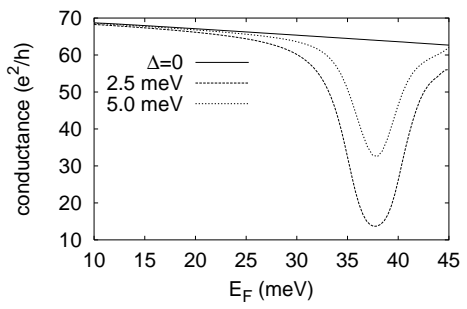

FIG. 1. Conductance as a function of Fermi energy for various coupling strengths at zero temperature and zero magnetic field. $\Delta=w k_{0}$ is the hybridization gap. Here $\hbar / \tau_{e}=0.8$ $\mathrm{meV}, \hbar / \tau_{h}=0.6 \mathrm{meV}$, and $E_{g 0}=50 \mathrm{meV}$.
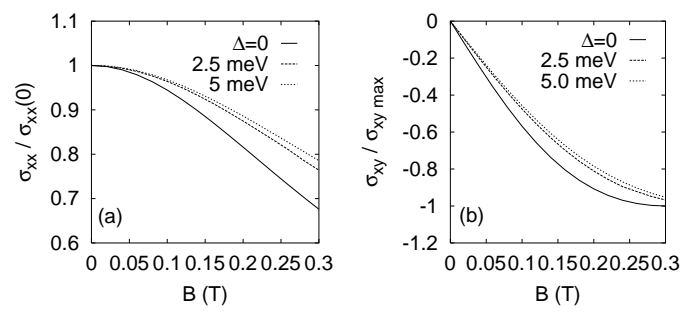

FIG. 2. (a) longitudinal and (b) transverse conductance as a function of magnetic field for the case when $E_{F}$ is at midgap and at zero temperature. $\sigma_{x y} \max$ is the maximum value of $\sigma_{x y}$ occurring at $B \approx 0.3-0.5$ T. $\tau_{e}, \tau_{h}$, and $E_{g 0}$ are as in Fig. 1.

Second, it is seen that magnetoconductance curves are affected by the presence of electron-hole coupling. This result may be of practical importance in the characterization of InAs-GaSb samples. That is because the usual characterization routines use sensitive fitting procedures of classical magnetoconductance expressions to measured data in order to extract four independent parameters: the electron and hole densities and mobilities [21]. The corrections to the classical expressions (i.e., to the case $\Delta=0$ ) due to hybridization may significantly affect the apparent value of these parameters.

In summary, we have studied the transport properties of coupled electron-holes within a full quantum approach. We arrived at a semi-analytic expression for the current under weak electric and magnetic fields, Eqs. (21,29). This current shows strong resistance resonance, does not vanish in the gap, and its magnetic-field dependence depends on the strength of coupling.

We are grateful to O. Agam, I. E. Aleiner, L. J. Cooper, N. K. Patel, M. Finkelstein, and L. D. Shvartsman for valuable discussions. The work in Jerusalem was supported by the Israel Science Foundation founded by the Israel Academy of Sciences and Humanities.

[1] M. Altarelli, Phys. Rev. B 28, 842 (1983).

[2] Y. Naveh and B. Laikhtman, Appl. Phys. Lett. 66, 1980 (1995).

[3] A. Palevski, F. Beltram, F. Capasso, L. Pfeiffer, and K. W. West, Phys. Rev. Lett. 65, 1929 (1990).

[4] F. T. Vasko, Phys. Rev. B 47, 2410 (1993).

[5] Y. Berk, A. Kamenev, A. Palevski, L. N. Pfeiffer, and D. W. West, Phys. Rev. B 50, 15420 (1994); ibid, 51, 2604 (1995).

[6] G. Gumbs and G. R. Aizin, Phys. Rev. B, 51, 7074 (1995).

[7] S. Das Sarma and E. H. Hwang, Phys. Rev. Lett. 81, 4216 (1998).

[8] J. J. Quinn and J. J. Quinn, Surf. Sci. 361/362, 930 (1996).

[9] S. de-Leon, L. D. Shvartsman, and B. Laikhtman, Phys. Rev. B 60, 1861 (1999).

[10] M.J. Yang, C.H. Yang, B.R. Bennett, and B.V. Shanabrook, Phys. Rev. Lett. 78, 4613 (1997).

[11] M. Lakrimi, S. Khym, R. J. Nicholas, D. M. Symons, F. M. Peeters, N. J. Mason, and P. J. Walker, Phys. Rev. Lett. 79, 3034 (1997).

[12] T. P. Marlow, L. J. Cooper, D. D. Arnone, N. K. Patel, D. M. Whittaker, E. H. Linfield, D. A. Ritchie, and M. Pepper, Phys. Rev. Lett. 82, 2362 (1999).

[13] A. J. L. Poulter, M. Lakrimi, R. J. Nicholas, N. J. Mason, and P. J. Walker, Phys. Rev. B 60, 1884 (1999).

[14] Yu. Vasilyev, S. Suchalkin, K. von Klitzing, B. Meltser, S. Ivanov, and P. Kop'ev, Phys. Rev. B 60, 10636 (1999).

[15] L.J. Cooper, N.K. Patel, V. Drouot, E.H. Linfield, D.A. Ritchie, and M. Pepper, Phys. Rev. B 57, 11915 (1998).

[16] M. J. Yang, C. H. Yang, and B. R. Bennett, Phys. Rev. B 60, R13958 (1999).

[17] L.D. Shvartsman, Solid State Comm. 46, 787 (1983).

[18] B. Laikhtman, S. de Leon, and L.D. Shvartsman, Solid State Comm. 104, 257 (1997).

[19] E. M. Lifshits, L. P. Pitaevskii, and L. D. Landau, Physical Kinetics (Pergamon, Oxford, 1981).

[20] B. Laikhtman and E. L. Altshuler, Ann. Phys. 232, 332 (1994).

[21] E.E. Mendez, L. Esaki, and L.L. Chang, Phys. Rev. Lett. 55, 2216 (1985). See also M.P. Mikhailova and A.N. Titkov, Semicond. Sci. Technol. 9, 1279 (1994) and references therein. 